\title{
Beamforming issues in modern MIMO Radars with Doppler
}

\author{
Chun-Yang Chen and P. P. Vaidyanathan \\ Dept. of Electrical Engineering, MC 136-93 \\ California Institute of Technology, Pasadena, CA 91125, USA \\ E-mail: cyc@caltech.edu,ppvnath@systems.caltech.edu
}

\begin{abstract}
In traditional beamforming radar systems, the transmitting antennas send coherent waveforms which form a highly focused beam. In the MIMO radar system, the transmitter sends noncoherent (possibly orthogonal) broad (possibly omnidirectional) waveforms. These waveforms can be extracted by a matched filterbank at the receiver. The extracted signals can be used to obtain more diversity or improve the clutter resolution.

This paper focuses on space-time adaptive processing (STAP) for MIMO radar systems which improves the clutter resolution. The size of the MIMO STAP steering vector can be much larger than the traditional SIMO STAP steering vector because of the extra dimension. An accurate estimation of clutter rank for the subspace method is developed, and is a generalization of Brennan's rule to the MIMO radar case. A data independent method for estimating the clutter subspace is also described. ${ }^{1}$
\end{abstract}

\section{INTRODUCTION}

Recently, the concept of MIMO radars has drawn considerable attention [1]- [10]. MIMO radars emit orthogonal waveforms [1]- [7] or noncoherent [8]- [10] waveforms instead of transmitting coherent waveforms which form a focused beam in the traditional transmit beamforming. In the MIMO radar receiver, a matched filterbank is used to extract the orthogonal waveform components. There are two major advantages of the system. First, increased spatial diversity can be obtained [3]. The orthogonal components are transmitted from different antennas. If these antennas are far enough from each other, the target radar cross sections (RCS) for different transmitting paths will become independent random variables. Thus each orthogonal waveform carries independent information about the target. This spatial diversity can be utilized to perform better detection [3]. Second, the phase differences caused by different transmitting antennas along with the phase differences caused by different receiving antennas can form a new virtual array steering vector. With judiciously designed antenna positions, one can create a very long, critically sampled, array steering vector at a small number of antennas. Thus the clutter resolution can be dramatically increased [1], [2] with a small cost. In this paper, we focus on this second advantage.

Adaptive techniques for processing the data from airborne antenna arrays are called space-time adaptive processing (STAP) techniques. The basic theory of STAP for the traditional SIMO radar has been well developed [18], [19]. There have been many algorithms proposed for improving the complexity and convergence of the STAP in the SIMO radar [18], [19]. With a slight modification, these methods can also be applied to the MIMO radar case. The MIMO extension to STAP can be found in [2]. However, in the MIMO radar, the space-time adaptive processing (STAP) becomes even more challenging because of the extra dimension created by the orthogonal waveforms. On the one hand, the

\footnotetext{
${ }^{1}$ Work supported in parts by the ONR grant N00014-06-1-0011, and the California Institute of Technology.
}

extra dimension increases the rank of the jammer and clutter subspace, especially the jammer subspace. This makes the STAP more complex. On the other hand, the extra degreeof-freedom created by the MIMO radar allows us to filter out more clutter subspace without affecting the SINR much. In this paper, we explore the clutter subspace and its rank in MIMO radar. The clutter rank in MIMO radar is estimated by a proposed rule. This can be viewed as an extension of Brennan's rule. Using the geometry of the MIMO radar and the prolate spheroidal wave functions (PSWF), a method for computing the clutter subspace is developed. The numerical example shows that under ideal condition (without ICM and array misalignment) the proposed clutter subspace estimation method is very accurate.

The rest of the paper is organized as follows. In Section II, the concept of MIMO radar will be briefly reviewed. In Section III, we formulate the STAP approach for MIMO radar. In Section IV, we explore the clutter subspace and its rank in the MIMO radar. Using prolate spheroidal wave function (PSWF), we are able to find a data independent basis for clutter signals. In Section $\mathrm{V}$, we test the proposed clutter subspace estimation method with a numerical example. Finally, Section VI concludes the paper.

Notations. Matrices are denoted by capital letters in boldface (e.g. A). Vectors are denoted by lowercase letters in boldface (e.g. x). Superscript ${ }^{\dagger}$ denotes transpose conjugation. The notation $\lceil a\rceil$ is defined as the smallest integer larger than a.

\section{REVIEW OF THE MIMO RADAR}

In this section, we briefly review the MIMO radar idea. More detailed reviews can be found in [1], [2], [4] We will focus on using MIMO radar to increase the degree-of-freedom. Fig. 1 illustrates a MIMO radar system. The transmitting an-
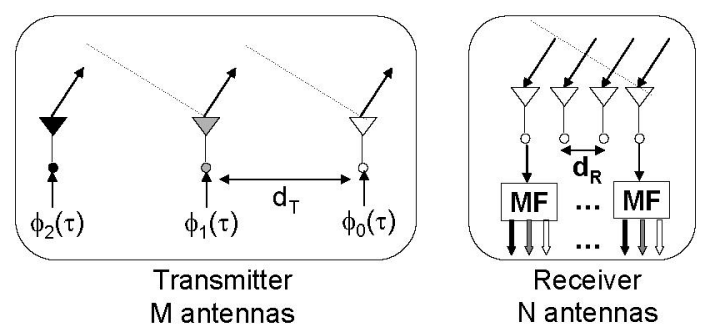

Fig. 1. Illustration of a MIMO radar system with $M=3$ and $N=4$.

tennas emit orthogonal waveforms. At each receiving antenna, these orthogonal waveforms can be extracted by $M$ matched 
filters, where $M$ is the number of transmitting antennas Therefore there are totally $N M$ extracted signals, where $N$ is the number of receiving antennas. The signals reflected by the target at direction $\theta$ can be expressed as

$$
\rho_{t} e^{j \frac{2 \pi}{\lambda}\left(n d_{R} \sin \theta+m d_{T} \sin \theta\right)},
$$

for $n=0,1, \cdots, N-1, m=0,1, \cdots, M-1$. Here $\rho_{t}$ is the amplitude of the signal reflected by the target, $d_{R}$ is the spacing between the receiving antennas, and $d_{T}$ is the spacing between the transmit antennas. The phase differences are created by both transmitting and receiving antenna locations. Define $f_{s} \triangleq\left(d_{R} / \lambda\right) \sin \theta$ and $\gamma \triangleq d_{T} / d_{R}$. Eq. (1) can be further simplified as

$$
\rho_{t} e^{j 2 \pi f_{s}(n+\gamma m)} .
$$

If we choose $\gamma=N$, the set $\{n+\gamma m\}=\{0,1, \cdots, N M-1\}$. Thus these $N M$ signals can be viewed as the signals received by a virtual array with $N M$ elements [2] as shown in Fig. II. It is as if we have a receiving array of $N M$ elements. Thus

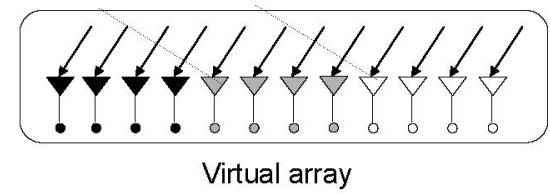

Fig. 2. The corresponding virtual array of the MIMO radar shown in Fig. 1.

a degree-of-freedom $N M$ can be obtained with only $N+M$ physical array elements. One can view the antenna array as a way to sample the electromagnetic wave in the spatial domain. The MIMO radar idea allows "sampling" in both transmitter and receiver and creates a total of $N M$ "samples". Taking advantage of these extra samples in spatial domain, a better clutter resolution can be obtained.

\section{STAP IN MIMO RADAR}

In this section, we formulate the STAP problem in MIMO radar. The MIMO extension for STAP first appeared in [2]. We will focus on the idea of using extra degree-of-freedom to increase the spatial resolution.

\section{A. Signal Model}

Fig. 3 shows the geometry of the MIMO radar STAP with uniform linear arrays (ULA), where

1) $d_{T}$ is the spacing of the transmitting antennas,

2) $d_{R}$ is the spacing of the receiver antennas,

3) $M$ is the number of transmitting antennas,

4) $N$ is the number of the receiving antennas,

5) $T$ is the radar pulse period,

6) $l$ indicates the index of radar pulse (slow time),

7) $\tau$ represents the time within the pulse (fast time),

8) $v_{t}$ is the target speed toward the radar station, and

9) $v$ is the speed of the radar station. (horizontal)

The radar station movement is assumed to be parallel to the linear antenna array. This assumption has been made in most of the airborne ground moving target indicator (GMTI) systems. Each array is composed of omnidirectional elements. The transmitted signals of the $m$ th antenna can be expressed as

$$
x_{m}(l T+\tau)=\sqrt{E} \phi_{m}(\tau) e^{j 2 \pi f(l T+\tau)}
$$

for $m=1,2, \cdots, M-1$, where $\phi_{m}(\tau)$ is the unmodulated waveform, $f$ is the carrier frequency, and $E$ is the transmitted energy for the pulse. The demodulated received signal of the $n$th antenna can be expressed as

$$
\begin{aligned}
& y_{n}\left(l T+\tau-\frac{2 r}{c}\right) \approx \\
& \sum_{m=0}^{M-1} \rho_{t} \phi_{m}(l T+\tau) e^{j \frac{2 \pi}{\lambda}\left(\sin \theta_{t}\left(2 v T l+d_{R} n+d_{T} m\right)+2 v_{t} T l\right)} \\
& +\sum_{i=0}^{N_{c}-1} \sum_{m=0}^{M-1} \rho_{i} \phi_{m}(l T+\tau) e^{j \frac{2 \pi}{\lambda}\left(\sin \theta_{i}\left(2 v T l+d_{R} n+d_{T} m\right)\right)} \\
& +y_{n}^{(J)}(l T+\tau)+y_{n}^{(w)}(l T+\tau),
\end{aligned}
$$

where

1) $r$ is the distance of the range bin of interest,

2) $c$ is the speed of light,

3) $\rho_{t}$ is the amplitude of the signal reflected by the target,

4) $\rho_{i}$ is the amplitude of the signal reflected by the $i$ th clutter,

5) $\theta_{t}$ is the looking direction of the target,

6) $\theta_{i}$ is the looking direction of the $i$ th clutter,

7) $N_{c}$ is the number of clutter signals,

8) $y_{n}^{(J)}$ is the jammer signal in the $n$th antenna, and

9) $y_{n}^{(w)}$ is the white noise in the $n$th antenna.

The first term in Eq. (2) represents the signal reflected by the target. The second term is the signal reflected by the clutter. The last line represents the jammer signal and white noise. We assume there is no internal clutter motion (ICM) or antenna array misalignment [18]. The phase differences in the reflected signals are caused by the Doppler shift, the differences of the receiving antenna locations, and the differences of the transmitting antenna locations. In the MIMO radar, the transmitting waveforms $\phi_{m}(\tau)$ satisfy orthogonality:

$$
\int \phi_{m}(\tau) \phi_{k}^{*}(\tau) d \tau=\delta_{m k}
$$

The sufficient statistics can be extracted by a set of matched filterbanks as shown in Fig. 3. The extracted signals can be expressed as

$$
\begin{aligned}
& y_{n, m, l} \triangleq \int y_{n}(l T+\tau-\Delta) \phi_{m}^{*}(\tau) d \tau= \\
& \rho_{t} e^{j \frac{2 \pi}{\lambda}\left(\sin \theta_{t}\left(2 v T l+d_{R} n+d_{T} m\right)+2 v_{t} T l\right)}+ \\
& \sum_{i=0}^{N_{c}-1} \rho_{i} e^{j \frac{2 \pi}{\lambda}\left(\sin \theta_{i}\left(2 v T l+d_{R} n+d_{T} m\right)\right)}+y_{n, m, l}^{(J)}+y_{n, m, l}^{(w)},
\end{aligned}
$$

for $n=0,1, \cdots, N-1, m=0,1, \cdots, M-1$, and $l=$ $0,1, \cdots, L-1$, where $y_{n, m, l}^{(J)}$ is the corresponding jammer signal, $y_{n, m, l}^{(w)}$ is the corresponding white noise, and $L$ is the number of the pulses in a coherent processing interval (CPI). To simplify the above equation, we define the following normalized spatial and Doppler frequencies:

$$
\begin{aligned}
f_{s} \triangleq \frac{d_{R}}{\lambda} \sin \theta_{t}, f_{s, i} \triangleq \frac{d_{R}}{\lambda} \sin \theta_{i} \\
f_{D} \triangleq \frac{2\left(v \sin \theta_{t}+v_{t}\right)}{\lambda} T .
\end{aligned}
$$

One can observe that the normalized Doppler frequency of the target is a function of both target looking direction and 


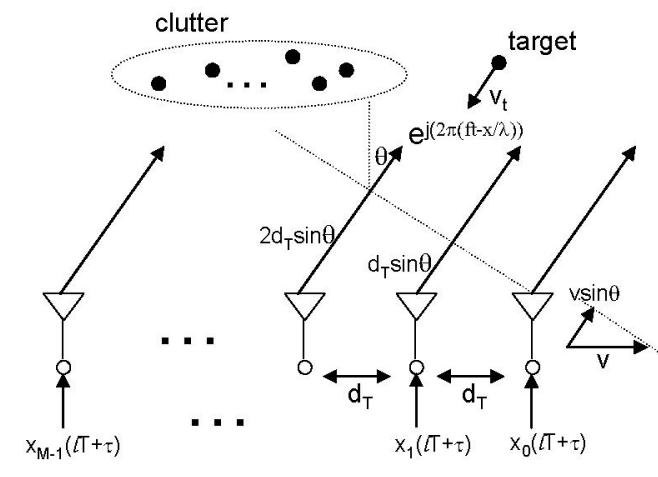

Transmitter

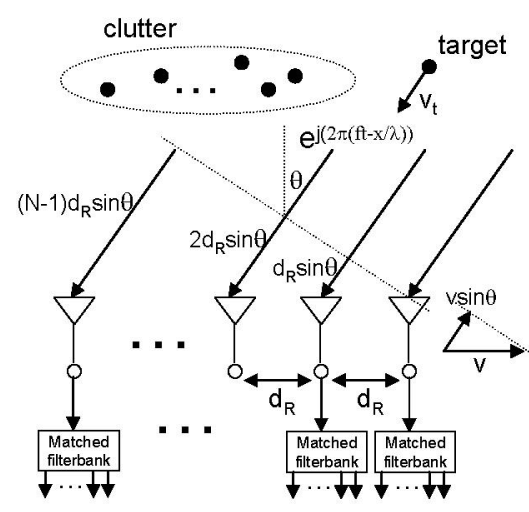

Receiver

Fig. 3. This figure illustrates a MIMO radar system with $M$ transmitting antennas and $N$ receiving antennas. The radar station is moving horizontally with speed $v$.

speed. Usually $d_{R}=\lambda / 2$ is chosen to avoid aliasing in spatial frequency. Using the above definition we can rewrite the extracted signal in Eq. (3) as

$$
\begin{aligned}
y_{n, m, l}= & \rho_{t} e^{j 2 \pi f_{s}(n+\gamma m)} e^{j 2 \pi f_{D} l}+ \\
& \sum_{i=0}^{N c-1} \rho_{i} e^{j 2 \pi f_{s, i}(n+\gamma m+\beta l)}+y_{n, m, l}^{(J)}+y_{n, m, l}^{(w)},
\end{aligned}
$$

for $n=0,1, \cdots, N-1, m=0,1, \cdots, M-1$, and $l=$ $0,1, \cdots, L-1$, where $\gamma \triangleq d_{T} / d_{R}$ and $\beta \triangleq 2 v T / d_{R}$.

\section{B. Fully adaptive MIMO-STAP}

The goal of space-time adaptive processing (STAP) is to find a linear combination of the extracted signals so that the SINR can be maximized. Thus the target signal can be extracted from the interferences, clutter, and noise to perform the detection. Stacking the MIMO STAP signals in Eq. (5), we obtain the $N M L$ vector

$$
\mathbf{y}=\left(\begin{array}{cccc}
y_{0,0,0} & y_{1,0,0} & \cdots & y_{N-1, M-1, L-1}
\end{array}\right)^{T} .
$$

Then the linear combination can be expressed as $\mathbf{w}^{\dagger} \mathbf{y}$, where $\mathbf{w}$ is the weighting for the linear combination. The SINR maximization can be obtained by minimizing the total variance under the constraint that the target response is unity. It can be expressed as the following optimization problem:

$$
\begin{aligned}
& \min _{\mathbf{w}} \mathbf{w}^{\dagger} \mathbf{R} \mathbf{w} \\
& \text { subject to } \mathbf{w}^{\dagger} \mathbf{s}\left(f_{s}, f_{D}\right)=1,
\end{aligned}
$$

where $\mathbf{R} \triangleq E\left[\mathbf{y y}^{\dagger}\right]$, and $\mathbf{s}\left(f_{s}, f_{D}\right)$ is the $N M L$ MIMO spacetime steering vector which consists of the elements

$$
e^{j 2 \pi f_{s}(n+\gamma m)} e^{j 2 \pi f_{D}},
$$

for $n=0,1, \cdots, N-1, m=0,1, \cdots, M-1$, and $l=0,1, \cdots, L-1$. This $\mathbf{w}$ is called minimum variance distortionless response (MVDR) beamformer. The covariance matrix $\mathbf{R}$ can be estimated by using the neighboring range bin cells. In practice, in order to prevent self-nulling, a target-free covariance matrix can be estimated by using guard cells [18]. The well-known solution to the above problem is [16]

$$
\mathbf{w}=\frac{\mathbf{R}^{-1} \mathbf{s}\left(f_{s}, f_{D}\right)}{\mathbf{s}\left(f_{s}, f_{D}\right)^{\dagger} \mathbf{R}^{-1} \mathbf{s}\left(f_{s}, f_{D}\right)} .
$$

However, the covariance matrix $\mathbf{R}$ is $N M L \times N M L$. It is much larger than in the SIMO case because of the extra dimension. The complexity of the inversion of such a large matrix is high. The estimation of such a large covariance matrix also converges slowly. To solve this problem, many partially adaptive techniques can be applied [18], [19]. These techniques often require estimation of the clutter-plus-jammer subspace or clutter subspace. The clutter subspace and its rank will be explored in the next section.

\section{Clutter Subspace of MiMO Radar Signals}

In this section, we explore the clutter subspace and its rank in the MIMO radar system. The covariance matrix $\mathbf{R}$ in $\mathrm{Eq}$. (7) can be expressed as $\mathbf{R}=\mathbf{R}_{t}+\mathbf{R}_{c}+\mathbf{R}_{J}+\sigma^{2} \mathbf{I}$, where $\mathbf{R}_{t}$ is the covariance matrix of the target signal, $\mathbf{R}_{c}$ is the covariance matrix of the clutter, $\mathbf{R}_{J}$ is the covariance matrix of the jammer, and $\sigma^{2}$ is the variance of the white noise. The clutter subspace is defined as the range space of $\mathbf{R}_{c}$ and the clutter rank is defined as the rank of $\mathbf{R}_{c}$. In the spacetime adaptive processing (STAP), it is well-known that the clutter subspace usually has a small rank. It was first pointed out by Klemm in [13], that the clutter rank is approximately $N+L$, where $N$ is the number of receiving antennas and $L$ is the number of pulses in a coherent processing interval (CPI) In [14], a rule for estimating the clutter rank was proposed. The estimated rank is approximately $N+\beta(L-1)$, where $\beta=2 v T / d_{R}$. It is called Brennan's rule. This result will now be extended to the MIMO radar.

\section{A. Clutter rank in MIMO radar}

We first study the clutter term in Eq. (5) which is expressed as

$$
y_{n, m, l}^{(c)}=\sum_{i=0}^{N c-1} \rho_{i} e^{j 2 \pi f_{s, i}(n+\gamma m+\beta l)}
$$

for $n=0,1, \cdots, N-1, m=0,1, \cdots, M-1$, and $l=$ $0,1, \cdots, L-1$. Note that $-0.5<f_{s, i}<0.5$ because $d_{R}=$ $\lambda / 2$. Define $c_{i, n, m, l}=e^{j 2 \pi f_{s, i}(n+\gamma m+\beta l)}$ and

$$
\mathbf{c}_{i}=\left(c_{i, 0,0,0}, c_{i, 1,0,0}, \cdots, c_{i, N-1, M-1, L-1}\right)^{T} .
$$


By stacking the signals $\left\{y_{n, m, l}^{(c)}\right\}$ into a vector, one can obtain

$$
\mathbf{y}^{(c)}=\sum_{i=0}^{N_{c}-1} \rho_{i} \mathbf{c}_{i} .
$$

Assume that $\rho_{i}$ are zero-mean independent random variables with variance $\sigma_{c, i}^{2}$. The the clutter covariance matrix can be expressed as

$$
\mathbf{R}_{c}=E\left[\mathbf{y}^{(c)} \mathbf{y}^{(c) \dagger}\right]=\sum_{i=0}^{N_{c}-1} \sigma_{c, i}^{2} \mathbf{c}_{i} \mathbf{c}_{i}^{\dagger} .
$$

Therefore, $\operatorname{span}\left(\mathbf{R}_{c}\right)=\operatorname{span}(\mathbf{C})$, where

$$
\mathbf{C} \triangleq\left(\mathbf{c}_{0}, \mathbf{c}_{1}, \cdots, \mathbf{c}_{N_{c}-1}\right) \text {. }
$$

The vector $\mathbf{c}_{i}$ consists of the samples of $e^{j 2 \pi f_{s, i} x}$ at points $\{n+\gamma m+\beta l\}$. In general, $\mathbf{c}_{i}$ is a nonuniformly sampled version of the bandlimited sinusoidal waveform $e^{j 2 \pi f_{s, i} x}$. If $\gamma$ and $\beta$ are both integers, the sampled points $\{n+\gamma m+\beta l\}$ can only be integers in

$$
\{0,1, \cdots, N+\gamma(M-1)+\beta(L-1)\} .
$$

If $N+\gamma(M-1)+\beta(L-1) \leq N M L$, there will be repetitions in the sample points. In other words, some of the row vectors in $\mathrm{C}$ will be exactly the same and there will be at most only $N+\gamma(M-1)+\beta(L-1)$ distinct row vectors in $\mathbf{C}$. Therefore the rank of $\mathbf{C}$ is less than $N+\gamma(M-1)+\beta(L-1)$. So is the rank of $\mathbf{R}_{c}$. We summarize this fact as the following theorem:

Theorem 1: If $\gamma$ and $\beta$ are both integers, than $\operatorname{rank}\left(\mathbf{R}_{c}\right) \leq$ $\min \left(N+\gamma(M-1)+\beta(L-1), N_{c}, N M L\right)$

Usually $N_{c}$ and $N M L$ are much larger then $N+\gamma(M-1)+$ $\beta(L-1)$. Therefore $N+\gamma(M-1)+\beta(L-1)$ is a good estimation of the clutter rank. This result can be viewed as a generalization of Brennan's rule [14] to the MIMO radar case.

Now we focus on the general case where $\gamma$ and $\beta$ are real numbers. The vector $\mathbf{c}_{i}$ in Eq. (10) can be viewed as a nonuniform sampled version of the truncated sinusoidal function

$$
c\left(f_{s, i}, x\right) \triangleq \begin{cases}e^{j 2 \pi f_{s, i} x}, & 0 \leq x \leq X \\ 0, & \text { otherwise }\end{cases}
$$

where $X \triangleq N-1+\gamma(M-1)+\beta(L-1)$. Furthermore, $-0.5 \leq$ $f_{s, i} \leq 0.5$ because $d_{R}$ is often selected as $\lambda / 2$ in Eq. (4) to avoid aliasing. Therefore, the energy of these signals is mostly confined to a constant time-frequency region. Such signals can be well approximated by linear combinations of $\lceil 2 W X+1\rceil$ orthogonal functions [15], where $W$ is the bandwidth and $X$ is the duration of the time-limited functions. In next section, more details on this will be discussed using prolate spheroidal wave function (PSWF). In this case, we have $W=0.5$ and $2 W X+1=N+\gamma(M-1)+\beta(L-1)$. The vectors $\mathbf{c}_{i}$ can be also approximated by the linear combination of the nonuniformly sampled versions of these $\lceil N+\gamma(M-1)+$ $\beta(L-1)\rceil$ orthogonal functions. Thus, in the case where $\gamma$ and $\beta$ are nonintegers, we can conclude that only $\lceil N+\gamma(M-$ 1) $+\beta(L-1)\rceil$ eigenvalues of the matrix $\mathbf{R}_{c}$ are significant In other words,

$$
\operatorname{rank}\left(\mathbf{R}_{c}\right) \approx\lceil N+\gamma(M-1)+\beta(L-1)\rceil .
$$

Note that the definition of this approximate rank is actually the number of the dominant eigenvalues. This notation has been widely used in the STAP literature.
The result can be further generalized for the array with arbitrary linear antenna deployment. Let $x_{T, m}, m=$ $0,1, \cdots, M-1$ be the transmitting antenna locations, $x_{R, n}, n=0,1, \cdots, N-1$ be the receiving antenna locations, and $v$ be the speed of the radar station. Without loss of generality, we set $x_{T, 0}=0$ and $x_{R, 0}=0$. Then the clutter signals can be expressed as

$$
y_{n, m, l}^{(c)}=\sum_{i=0}^{N_{c}-1} \rho_{i} e^{j \frac{2 \pi}{\lambda} \sin \theta_{i}\left(\left(x_{R, n}+x_{T, m}+2 v T l\right)\right)}
$$

for $n=0,1, \cdots, N-1, m=0,1, \cdots, M-1$, and $l=$ $0,1, \cdots, L-1$, where $\theta_{i}$ is the looking-direction of the $i$ th clutter. The term

$$
e^{j \frac{2 \pi}{\lambda} \sin \theta_{i}\left(x_{R, n}+x_{T, m}+2 v T l\right)}
$$

can also be viewed as a nonuniform sampled version of the function $e^{j \frac{2 \pi}{\lambda} \sin \theta_{i} x}$. Using the same argument we have made in the uniform linear array (ULA) case, one can obtain

$$
\operatorname{rank}\left(\mathbf{R}_{c}\right) \approx\left\lceil 1+\frac{2}{\lambda}\left(x_{R, N-1}+x_{T, M-1}+2 v T(L-1)\right)\right\rceil .
$$

One can see that the number of dominant eigenvalues is proportional to the ratio of the total aperture of the space-time virtual array and the wavelength.

\section{$B$. Data independent estimation of the clutter subspace with PSWF}

The clutter rank can be estimated by using Eq. (12) and the parameters $N, M, L, \beta$ and $\gamma$. However, the clutter subspace is often estimated by using data samples. In this section, we propose a method which estimates the clutter subspace using the geometry of the problem rather than the received signal. The main advantage of this method is that it is data independent. Therefore the corresponding STAP method converges faster than the data dependent methods. Experiments also show that the estimated subspace is very accurate in the ideal case (without ICM and array misalignment).

The signal in Eq. (11) is time-limited and most of its energy is concentrated on $-0.5 \leq f_{s} \leq 0.5$. To approximate the subspace which contains such signals, we find the basis functions which are time-limited and concentrate their energy on the corresponding bandwidth. Such basis functions are the solutions of the following integral equation [15]

$$
\mu \psi(x)=\int_{0}^{X} \operatorname{sinc}(2 W(x-\zeta)) \psi(\zeta) d \zeta,
$$

where $\operatorname{sinc}(x) \triangleq \frac{\sin \pi x}{\pi x}$ and $\mu$ is a scalar to be solved. This integral equation has infinite number of solutions $\psi_{i}(x)$ and $\mu_{i}$ for $i=0,1, \cdots, \infty$. The solution $\psi_{i}(x)$ is called prolate spheroidal wave function (PSWF). By the maximum principle [20], the solution satisfies

$$
\begin{gathered}
\psi_{0}(x)=\arg \max _{\|\psi\|=1} \int_{0}^{X} \int_{0}^{X} \psi^{*}(x) \operatorname{sinc}(2 W(x-\zeta)) \psi(\zeta) d \zeta d x \\
\psi_{i}(x)=\arg \max _{\|\psi\|=1} \int_{0}^{X} \int_{0}^{X} \psi^{*}(x) \operatorname{sinc}(2 W(x-\zeta)) \psi(\zeta) d \zeta d x \\
\text { subject to } \int_{0}^{X} \psi(x) \psi_{k}^{*}(x) d x=0, \text { for } k=0,1, \cdots, i-1,
\end{gathered}
$$

for $i=1,2, \cdots, \infty$. The function $\psi_{i}(x)$ is orthogonal to the previous basis components $\psi_{k}(x)$, for $k<i$ while concentrating most of its energy on the bandwidth $[-W, W]$. Moreover, 
only the first $[2 W X+1\rceil$ eigenvalues $\mu_{i}$ are significant [15]. Therefore, the time-band-limited function $c\left(f_{s, i}, x\right)$ in Eq. (11) can be well approximated by linear combinations of $\psi_{i}(x)$ for $i=0,1, \cdots,\lceil 2 W X+1\rceil$. In this case, $W=0.5$ and $2 W X+1=N+\gamma(M-1)+\beta(L-1)$. Thus the nonuniformly sampled version of $c\left(f_{s, i}, x\right)$, namely $c_{i, n, m, l}$, can be approximated by the linear combination:

$c_{i, n, m, l} \triangleq e^{j 2 \pi f_{s, i}(n+\gamma m+\beta l)} \approx \sum_{k=0}^{r_{c}-1} \alpha_{i, k} \psi_{k}(n+\gamma m+\beta l)$,

for some $\left\{\alpha_{i, k}\right\}$ where $r_{c} \triangleq\lceil N+\gamma(M-1)+\beta(L-1)\rceil$. Stacking the above elements into vectors, we have

$$
\mathbf{c}_{i} \approx \sum_{k=0}^{r_{c}-1} \alpha_{i, k} \mathbf{u}_{k}
$$

where $\mathbf{u}_{k}$ is a vector which consists of the elements $\psi_{k}(n+$ $\gamma m+\beta l$ ). Finally, we have

$$
\operatorname{span}\left(\mathbf{R}_{c}\right)=\operatorname{span}(\mathbf{C}) \approx \operatorname{span}\left(\mathbf{U}_{c}\right),
$$

where $\mathbf{U}_{c} \triangleq\left(\begin{array}{llll}\mathbf{u}_{0} & \mathbf{u}_{1} & \cdots & \mathbf{u}_{r_{c}-1}\end{array}\right)$. In practice, the PSWF $\psi_{i}(x)$ can be computed off-line and stored in the memory. When the parameters change, one can obtain the vectors $\mathbf{u}_{k}$ by resampling the PSWF $\psi_{k}(n+\gamma m+\beta l)$ to form the new clutter subspace.

\section{NUMERICAL EXAMPLES}

In this section, the accuracy of the clutter subspace estimation method is demonstrated by a numerical example. Performing the Gram-Schmidt procedure on the basis $\left\{\mathbf{u}_{k}\right\}$, we obtain the orthonormal basis $\left\{\mathbf{q}_{k}\right\}$. The clutter power in each orthonormal basis element can be expressed as $\mathbf{q}_{k}^{\dagger} \mathbf{R}_{c} \mathbf{q}_{k}$. Fig. 4 shows the clutter power in the orthogonalized basis elements. In this example, $N=10, M=5, L=16, \gamma=10$,

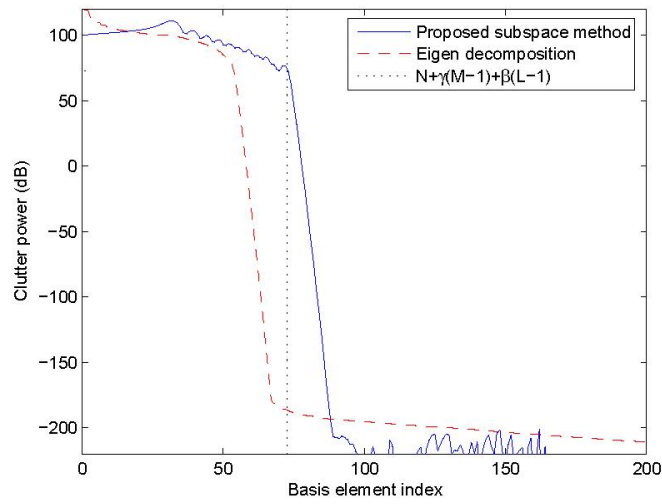

Fig. 4. Plot of the clutter power distributed in each of the orthogonal basis elements.

and $\beta=1.5$. The latitude is $9 \mathrm{~km}$ and the range of interest is $12.728 \mathrm{~km}$. For this latitude and range, the clutter is generated by using the model in [12]. The clutter to noise ratio (CNR) is $40 \mathrm{~dB}$. Note that there are totally $N M L=800$ basis elements but we only show the first 200 on the plot. The eigenvalues of $\mathbf{R}_{c}$ are also shown in Fig. 4 for comparison (red). The estimated clutter rank is $[N+\gamma(M-1)+\beta(L-1)\rceil=73$. One can see that the proposed subspace method (blue) is very accurate. The subspace captures almost all clutter power. Compared to the eigen decomposition method, the subspace obtained by the new method is larger. This is because for some range bins, the clutter looking direction is limited. However, the method has the advantage that it is data independent and can be computed off-line.

\section{Conclusions}

In this paper, we explored how to capture the clutter subspace and its rank in MIMO radars using the geometry of the system. The rule for estimating the clutter rank was extended to MIMO radars. An algorithm for computing the clutter subspace using nonuniform sampled PSWF was described. The numerical example shows that the proposed clutter subspace estimation method is very accurate. The proposed method can be used in a STAP method. The corresponding result has been submitted [17].

In this paper, we only consider the ideal case. In fact, the clutter subspace might change because of effects such as the internal clutter motion (ICM) or velocity misalignment [18]. In this case, a better way might be estimating the clutter subspace by using a combination of both the geometry and the received data. This idea will be explored in the future.

\section{REFERENCES}

[1] D. J. Rabideau and P. Parker, "Ubiquitous MIMO Multifunction Digital Array Radar," Proc. 37th IEEE Asil. Conf. on Signals, Systems, and Computers, pp. 1057-1064, Nov. 2003.

[2] D. W. Bliss and K. W. Forsythe, "Multiple-input multiple-output (MIMO) radar and imaging: degrees of freedom and resolution," Proc. 37th IEEE Asil. Conf. on Signals, Systems, and Computers, pp. 54-59, Nov. 2003.

[3] E. Fishler, A. Haimovich, R. S. Blum, L. J. Cimini, D. Chizhik, and R. A. Valenzuela, "Spactial Diversity in Radars-Models and Detection Performance," IEEE Trans. Sig. Proc., pp. 823-837, March 2006.

[4] F. C. Robey, S. Coutts, D. Weikle, J. C. McHarg, and K. Cuomo, "MIMO Radar Theory and Experimental Results," Proc. 38th IEEE Asil. Conf. on Signals, Systems, and Computers, pp. 300-304, Nov. 2004.

[5] K. W. Forsythe, D. W. Bliss, and G. S. Fawcett, "Multiple-Input MultipleOutput (MIMO) Radar Performance Issues," Proc. 38th IEEE Asil. Conf. on Signals, Systems, and Computers, pp. 310-315, Nov. 2004.

[6] H. A. Khan and D. J. Edwards, "Doppler problems in orthogonal MIMO radars," IEEE International Radar Conference, pp. 24-27, April 2006.

[7] V. F. Mecca, D. Ramakrishnan, and J. L. Krolik, "MIMO Radar SpaceTime Adaptive Processing for Multipath Clutter Mitigation" IEEE Workshop SAM, pp. 249-253, July 2006.

[8] D. R. Fuhrmann and G. S. Antonio, "Transmit Beamforming for MIMO Radar Systems Using Partial Signal Correlation," Proc. 38th IEEE Asil. Conf. on Signals, Systems, and Computers, pp. 295-299, Nov. 2004.

[9] G. S. Antonio and D. R. Fuhrmann, "Beampattern Synthesis for Wideband MIMO Radar Systems," Proc. 1st. IEEE Workshop CAMSAP, pp. 105108, Dec. 2005.

[10] K. W. Forsythe and D. W. Bliss, "Waveform Correlation and Optimization Issues for MIMO Radar," Proc. 39th IEEE Asil. Conf. on Signals, Systems, and Computers, pp. 1306-1310, Nov. 2005

[11] Q. Zhang and W. B. Mikhael, "Estimation of The Clutter Rank in the Case of Subarraying for Space-Time Adaptive Processing," Electronics Letters, pp. 419-420, 27 Feb 1997.

[12] N. A. Goodman and J.M. Stiles, "On Clutter Rank Observed by Arbitrary Arrays," accepted to IEEE Trans. on Signal Processing.

[13] R. Klemm, "Adaptive clutter supression for airborne phased array radars", IEE Proc. F, 1983, 130, (1), pp. 125-132.

[14] J. Ward, "Space-Time Adaptive Processing for Airborne Radar," Technical Report 1015, Lincoln Laboratory, Dec. 1994

[15] D. Slepian, and H. O. Pollak, "Prolate Spheroidal Wave Functions, Fourier Analysis and Uncertainty-III: the dimension of the space of essentially time-and-band-limited signals," Bell Syst. Tech. J., pp. 1295 1336, July 1962.

[16] J. Capon, "High-resolution frequency-wavenumber spectrum analysis," Proc. IEEE, vol. 57, no. 8, pp. 1408-1418, Aug. 1969.

[17] C. Y. Chen and P. P. Vaidyanathan, "A Subspace Method for MIMO Radar Space-Time Adaptive Processing," Submitted to ICASSP 2007.

[18] J. R. Guerci, Space-time adaptive processing, Artech House, 2003.

[19] R. Klemm, Principles of Space-Time Adaptive Processing, IEE, 2002.

[20] J. P. Keener, Principles of Applied Mathematics, Addison-Welsley, 1988. 